\title{
Laparoscopic partial nephrectomy: The McMaster University experience
}

\author{
Naji J. Touma, MD, FRCSC; ${ }^{*}$ Edward D. Matsumoto, MD, FRCSC; ${ }^{\dagger}$ Anil Kapoor, MD, FRCSC ${ }^{\dagger}$ \\ *Department of Urology, Queen's University, Kingston, ON; 'Division of Urology, McMaster University, Hamilton, ON
}

Cite as: Can Urol Assoc J 2012;6(4):233-7. hitp://dx.doi.org/10.5489/cuaj.11256

\begin{abstract}
Introduction: Laparoscopic partial nephrectomy (LPN) remains one of the more challenging procedures in urology. Minimizing warm ischemia time (WIT) and bleeding requires efficient intracorporeal suturing. In addition, achieving negative surgical margins requires complete excision of the tumour. We report a large Canadian series of laparoscopic partial nephrectomy with intermediate follow-up. Methods: Between September 2000 and August 2008, 152 consecutive laparoscopic partial nephrectomies were performed at our centre. Demographic, pathological and clinical data were collected through a retrospective review of the charts.

Results: The average tumour size was $2.68 \mathrm{~cm}$ (Range: 0.5-8.8. The vast majority of tumours were malignant $(80 \%)$. All margins were negative, except for 2 patients who underwent an immediate re-resection. There were no local recurrences or distant metastasis during the follow-up period of 44.3 months. Most procedures required hilar clamping (93.4\%) with a mean WIT of 34 minutes, with a clear trend for declining WIT with increasing experience. Five procedures were converted to laparoscopic radical nephrectomy, 10 converted to a hand-assisted procedure, and 1 was converted to an open partial nephrectomy. The average blood loss was $162 \mathrm{cc}$. Complications related to the procedure were classified according to the Clavien grading system. The average drop in the glomerular filtration rate was calculated by the Modification of Diet in Renal Disease (MDRD) Study equation between preoperative and 2.5 months postoperative was $8.6 \mathrm{~mL} / \mathrm{min} / 1.73 \mathrm{~m}^{2}$.

Conclusions: LPN is a challenging procedure that requires advanced laparoscopic skills. LPN is feasible with excellent oncological outcomes, and an acceptable complication profile. The short-term impact on overall renal function is minimal. The most common postoperative complication was pseudo-aneurysm requiring embolization, which reinforces the intra-operative need for meticulous and a quick suture-ligation of blood vessels during LPN.
\end{abstract}

\section{Introduction}

In 2008, renal cell carcinoma (RCC) was the ninth most common cancer in Canada, with 4400 new cases diag- nosed. ${ }^{1}$ Surgical extirpation has been the mainstay of RCC treatment since the work of Robson and colleagues four decades ago. ${ }^{2}$ The techniques have evolved dramatically since then with nephron-sparing surgery ${ }^{3}$ and laparoscopy. ${ }^{4}$ Over the last 20 years, two trends in RCC have emerged: a decrease in the size of diagnosed tumours and a migration towards lower stages. ${ }^{5}$ At the same time, an emerging body of literature is strengthening the case for partial nephrectomy (PN) as the treatment of choice for small renal masses. PN has been shown to be equivalent to radical nephrectomy (RN) for renal masses $<4 \mathrm{~cm}$ in terms of 10 -year cancerspecific survival rates. ${ }^{6}$ Recently, a few series have even reported the oncologic equivalency of $\mathrm{PN}$ and $\mathrm{RN}$ for masses between 4 and $7 \mathrm{~cm}^{7,8}$ In addition, PN has been shown to be superior to RN in terms of renal function preservation. ${ }^{9,10}$

Despite strong support for $\mathrm{PN}$ as the procedure of choice for the treatment of small renal masses, it remains significantly underutilized. In a review of the Surveillance, Epidemiology and End Results (SEER) registry, Miller and colleagues reported that only $9.6 \%$ of nephrectomies performed between 1988 and 2001 were partial. ${ }^{11}$ This underuse may be due to an unfamiliarity with the techniques of an open partial nephrectomy (OPN). This problem will only be exacerbated as centres of excellence establish laparoscopy as the new standard approach for PN. Laparoscopic partial nephrectomy (LPN) is the most demanding of the laparoscopic procedures due to the potential for hemorrhage, the time pressure of warm ischemia, the need for efficient intracorporeal suturing and the need for complete ease and comfort with the procedure.

At McMaster University, Hamilton, Ontario, LPN has been performed for small renal masses since 2000, mostly by one surgeon (AK). With time, there has been accumulation of expertise, and this series is currently one of the larger Canadian ones. We reviewed whether warm ischemia times (WITs) declined with increased experience and whether the use of LPN increased for more challenging tumours (i.e., tumour size). To justify the use of tumour size as a surrogate 
Touma et al.

for degree of difficulty of LPN, a correlation between tumour size and WIT was analyzed. Nephrometry scores (R.E.N.A.L or PADUA) were not available due to the lack of availability of imaging in order to be able to assign the scores retrospectively. In this retrospective analysis, the intermediate-term oncologic control of this procedure, as well as the decline in renal function over the short term following LPN, was reviewed. Finally, surgical complications related to LPN were tabulated.

\section{Methods}

Between September 2000 and August 2008, 152 LPNs were performed. Most cases (88.2\%) were performed by one surgeon (AK), who performed PNs exclusively through a laparoscopic approach. While an open approach was performed by the three other participating surgeons, the selection criteria for the LPN for the minority of the overall series $(11.8 \%)$ were largely based on each surgeon's judgment. Through a retrospective review of the patient charts, we collected demographic, pathological and clinical data. The estimated glomerular filtration rate (GFR) was calculated using the modification of diet in renal disease (MDRD) study equation. $^{12}$ The postoperative GFR values were calculated using the first available outpatient values, which was on average 2.5 months postoperatively. Changes between preeoperative and postoperative proportions in each chronic kidney disease (CKD) category were compared using the Fisher's exact test. Tumour size was collected from computed tomography scan reports as the greatest measured dimension prior to the procedure. Plots were then drawn of the change in tumour size and WIT time over the length of time of the series. To determine if tumour size constituted a valid surrogate of the degree of difficulty of LPN, a plot of tumour size versus warm ischemia time was also drawn. Correlations between the different variables were verified using a linear regression model.

Our surgical technique for LPN has evolved over time. Through a transperitoneal approach, the bowel was dissected along the white line of Toldt, exposing the retroperitoneum, and later the renal hilum. Gerota's fascia was opened to expose the tumour. A laparascopic ultrasound was used only to localize endophytic tumours. Vascular control was obtained with a laparoscopic DeBakey (Scanlan International, Inc., Saint Paul, MN) or Satinsky (Karl Storz, Tuttlingen, Germany) clamps. Initially, the entire hilum was clamped, but later in the series, only the renal artery was controlled. In a minority of cases, hand-assisted compression was used for vascular control. Lastly, a few cases, usually smaller renal masses, were done without any vascular control. Laparoscopic scissors were used without thermal energy for dissection of the renal mass. Frozen sections were not sent on a routine basis. If the collecting system was entered, this was repaired with 4-0 vicryl sutures, as were all visible blood vessels. A gelfoam or oxycel bolster would then be inserted into the parenchymal defect and the parenchyma would be brought together with the help of Lapra-Ty (Ethicon Endosurgery, Cincinnati, OH.) Sealing agents were used at the discretion of the surgeon.

\section{Results}

The median patient age was 60 years; a little over half of our patients were male $(57.6 \%)$. The median combined condition and age-related Charlson comorbidity score was 3. A review of the pathology reports revealed that $80 \%$ of the resected lesions were malignant and $20 \%$ were benign. Of the malignant lesions, $68 \%$ were clear cell RCC, 22\% papillary RCC, $8 \%$ chromophobe RCC and $2 \%$ or one lesion was a lymphoma. Of the benign tumours, 44\% were benign cysts, $24 \%$ angiomyolipomas, $20 \%$ oncocytomas and $12 \%$ were miscellaneous (i.e., other benign lesions, including adenoma, hemangioma and leiomyoma). Three patients had functionally solitary kidneys at the time of LPN, including two with contralateral atrophic kidneys and one previous laparoscopic nephrectomy for RCC. One patient had previously undergone an OPN for a contralateral RCC. The mean tumour size was $2.68 \mathrm{~cm}$ (range: 0.5-8.8). The classification of tumours was as follows: $89 \%$ of the tumours were clinical stage $\mathrm{T} 1 \mathrm{a} ; 10.4 \% \mathrm{~T} 1 \mathrm{~b} ;$ and $0.6 \% \mathrm{~T} 2$. There was a slight increase in the mean tumour size over the eight years that were analyzed. There was also a significant increase in the amount of LPNs that were performed from 2000 to 2008. Over the eight-year period, the average WIT decreased and a positive correlation was found between WIT and tumour size. This latest correlation demonstrates that increasing tumour size leads to increased difficulty of the critical part of LPN (i.e., tumour resection and defect repair).

The mean patient follow-up time was 44.3 months. Two patients $(1.3 \%)$ had grossly positive margins, which were resected immediately. There were no local recurrences or metastases during the follow-up period.

Most of the LPNs (93.40\%) were performed using a clamp; the average clamp time was 34 minutes (range: 7-58). Pelvicalyceal repair was necessary in $19.7 \%$ of cases. Of the 152 cases, $89.5 \%$ were completed as LPN, 6.6\% were converted to a hand-assisted procedure and $0.6 \%$ was converted to an OPN. In 3.3\% of the cases, a laparoscopic RN was performed because a PN was deemed not feasible by the surgeon after the renal exploration.

The median operative time was 3.5 hours and the median length of hospital stay was 4 days. The mean estimated blood loss was 162 cc and the average drop in hemoglobin was $29 \mathrm{~g} / \mathrm{L}$ between the preoperative and the postoperative day 1 values. The overall complication rate was $13.8 \%$, including one postoperative death secondary to a pulmonary embolus. 
The surgical complications were broken down according to the Clavien grading system (Table 1). ${ }^{13}$ This overall rate was an underestimate of minor complications as the record keeping was not deemed accurate enough to retrospectively and accurately tabulate all Clavien grade 1 complications.

Change in renal function before and after surgery was available for 70 patients. Using the preoperative and postoperative calculated GFR, the patients' CKD was determined using the classification system established by National Kidney Foundation's Kidney Disease Outcome Quality Initiative (K/DOOI) advisory board. ${ }^{14}$ The mean drop in GFR was $8.6 \mathrm{~mL} / \mathrm{min} / 1.73 \mathrm{~m}^{2}$, with none of the patients requiring postoperative dialysis. There was a slight shift with fewer patients falling into stage 1 CKD postoperatively (GFR $>90 \mathrm{~mL} / \mathrm{min} / 1.73 \mathrm{~m}^{2}$ ), and more falling into stage 3 CKD $\left.\left(30 \mathrm{~mL} / \mathrm{min} / 1.73 \mathrm{~m}^{2}\right)<\mathrm{GFR}<60 \mathrm{~mL} / \mathrm{min} / 1.73 \mathrm{~m}^{2}\right)$. However, these shifts were not statistical significant.

\section{Discussion}

Absolute indications for PN include a tumour in a solitary kidney, compromised renal function and a hereditary disorder that predisposes to recurrent RCC. However, the value of elective PN is being increasingly shown in studies demonstrating oncologic equivalency to $\mathrm{RN}$ in stage $\mathrm{T} 1$ tumours, ${ }^{7,8}$ as well as superiority in renal function preservation. ${ }^{9,10}$ Huang and colleagues reviewed patients with renal tumours $<4 \mathrm{~cm}$ and normal renal function. They demonstrated that those who underwent a PN had a much reduced risk of developing CKD compared to those undergoing a RN during the follow-up period. ${ }^{9}$ Similarly, Lau and colleagues matched a cohort of patients for stage, grade, age, sex, tumour size and year of surgery. They demonstrated the superiority of $\mathrm{PN}$ over $\mathrm{RN}$ in preserving renal function in patients with unilateral RCC and a normal contralateral kidney. ${ }^{10}$ The importance of renal function preservation resides not only in preventing end-stage renal disease and dialysis, but also in preventing CKD. CKD is defined as a GFR $<60 \mathrm{~mL} / \mathrm{min} / 1.73 \mathrm{~m}^{2}$, and has been demonstrated to be an independent risk factor for cardiovascular disease, hospitalization and death. ${ }^{15,16}$ Consequently, it has been speculated that overuse of $\mathrm{RN}$ for tumours that are otherwise amenable for nephron sparing may lead to increased non-cancer specific mortality. In fact, Huang and colleagues examined the SEER registry for patients over 66 years old with RCC $<4 \mathrm{~cm}$ treated with RN or PN. They concluded that $\mathrm{RN}$ treatment places these patients at increased risk for cardiovascular events and overall mortality. ${ }^{17}$

It is increasingly evident that PN should be the treatment of choice for small renal masses. In fact, the American Urological Association guidelines place PN as standard treatment for all T1a lesions, as well as an alternate standard to $\mathrm{RN}$ for T1b lesions in otherwise healthy patients. ${ }^{18}$ However, it is unclear whether these results are reproducible with the laparoscopic approach. Beyond the minimally-invasive approach, some major differences distinguish LPN from OPN. Firstly, LPN is mostly performed transperitoneally, whereas a retroperitoneal approach is preferred for OPN. Secondly, cooling is often cumbersome and rarely used in LPN, compared to OPN where it is usually applied. Lastly, hemostatic agents are more widely used in LPN, whereas OPN relies more on suturing of individual vessels. The largest series comparing LPN and OPN examined $1800 \mathrm{PNs}$ performed at the Cleveland clinic, Johns Hopkins and the Mayo clinic. ${ }^{19}$ OPN patients were more likely to present symptomatically, have a decreased performance status and have impaired renal function or a solitary kidney. In addition, tumours in the OPN group were larger, more centrally located and more likely to be malignant. The advantages of LPN were shorter operative time, decreased blood loss and decreased hospital stay. The advantages of OPN were decreased ischemia time, decreased postoperative complications and a decreased number of subsequent procedures. The two approaches were equivalent for three-year cancerspecific survival and renal function at three months.

Increasing tumour size seems to add to the complexity of a PN. Gill and colleagues have shown that $8.8 \%$ of LPN tumours were $\mathrm{T} 1 \mathrm{~b}$, compared to $31.4 \%$ of OPN cases. ${ }^{19}$ In addition, OPN for tumours $>4 \mathrm{~cm}$ have been shown to be associated with increased operative time, blood loss, transfusions and urinary fistula. ${ }^{20}$ Data on the impact of tumour size on outcomes in LPN are lacking. We have shown that increased tumour size is associated with increased WIT in LPN. Whether this increased WIT translates into any longterm impact on renal function remains to be seen. In addition, the mean WIT in this series was 34 minutes. There was, however, a clear trend for a decline in WIT with increasing experience. In a review of the current literature, it would appear that WIT in LPN are consistently longer than OPN. ${ }^{21}$ It has been speculated that given the increased abdominal pressure in laparoscopy leading to oliguria, there is a preconditioning of the renal unit to tolerate increased WIT in LPN. This, however, remains controversial and very little comparative data exists to distinguish the impact on renal

\begin{tabular}{|c|c|c|}
\hline Grade & Total no. & Surgical complications (n) \\
\hline Grade 2 & 12 & $\begin{array}{c}\text { Transfusion (9), myocardial infarction (2), } \\
\text { pulmonary embolus (1) }\end{array}$ \\
\hline Grade 3a & 2 & Pseudoaneurysm requiring embolization (2) \\
\hline Grade 3b & 4 & $\begin{array}{c}\text { Postoperative exploration for bleed (2), } \\
\text { urine leak requiring stent (1), transected } \\
\text { ureter requiring intra-operative uretero- } \\
\text { ureterostomy (1) }\end{array}$ \\
\hline Grade 4 & 2 & Cardiogenic shock (1), sepsis (1) \\
\hline Grade 5 & 1 & Death from a pulmonary embolus \\
\hline
\end{tabular}


Touma et al.

function of OPN and LPN. In this series, we demonstrated a modest impact on renal function after LPN.

Finally, the oncologic control obtained with LPN compares favourably with OPN over the intermediate term. The local recurrence rates and cancer specific survival appeared to be similar with both techniques. ${ }^{21}$ It is noteworthy, however, that most OPN series are quite mature, whereas only the Cleveland clinic has reported five-year cancer-specific survivals in a limited number of patients. ${ }^{22}$ In this series, no incidence of local or systemic recurrence has been noted over a 44.3 months follow-up period.

\section{Conclusion}

LPN is a challenging procedure with a significant learning curve. However, there are significant advantages to the use of this procedure over OPN for small renal masses. While LPN has increased warm ischemia times compared to OPN, it offers shorter recovery time, less analgesic use and improved quality of life for patients. Early oncological outcomes show equivalency to OPN, but long-term renal function follow-up for LPN patients is lacking. It should be stressed, however, that OPN remains a viable option for nephron sparing, and should not be substituted with a laparoscopic radical nephrectomy when the skills or expertise are lacking to perform LPN. While this study demonstrates that LPN is a feasible option for small renal masses, its limitations should be stressed. This is a single institution experience of largely one surgeon. Careful patient and tumour selection criteria should be applied, especially early in any surgeon's experience with LPN.

Competing interests: None declared.

This paper has been peer-reviewed.

\section{References}

1. Canadian Cancer Society/National Cancer Institute of Canada. Canadian Cancer Statistics 2008. Toronto, Canada, 2008.

2. Robson CJ, Churchill BM, Anderson W. The results of radical nephrectomy for renal cell carcinoma. J Urol 1969;101:297-301.
3. Novick AC, Stewart BH, Straffon RA, et al. Partial nephrectomy in the treatment of renal adenocarcinoma. J Urol 1977; 118:932-6.

4. Clayman RV, Kavoussi LR, Soper NJ, et al. Laparoscopic nephrectomy: Initial case report. J Urol 1991;146:278-82.

5. Copperberg MR, Mallin K, Ritchey J, et al. Decreasing size at diagnosis of stage 1 renal cell carcinoma: analysis from the national cancer data base, 1993 to 2004. J Urol 2008; 179:2131-5. http://dx.doi. org/10.1016/i.juro.2008.01.097

6. Fergany AF, Hafez KS, Novick AC. Long-term results of nephron sparing surgery for localized renal cell carcinoma: 10-year follow-up. J Urol 2000;163:442-5. http://dx.doi.org/10.1016/S0022$5347(05) 67896-2$

7. Leibovich BC, Blute ML, Cheville JC, et al. Nephron sparing surgery for appropriately selected renal cell carcinoma between 4 and $7 \mathrm{~cm}$ results in outcome similar to radical nephrectomy. J Urol 2004;171:106670. http://dx.doi.org/10.1097/01.ju.0000113274.40885.db

8. Pahernik $S$, Roos F, Röhrig $B$, et al. Elective nephron sparing surgery for renal cell carcinoma larger than 4 cm. J Urol 2008;179:71-4. http://dx.doi.org/10.1016/i.juro.2007.08.165

9. Huang WC, Levey AS, Serio AM, et al. Chronic kidney disease after nephrectomy in patients with renal cortical tumours: a retrospective cohort study. Lancet Oncol 2006;7:735-40. hitp://dx.doi.org/10.1016/ S1470-2045(06)70803-8

10. Lau WKO, Blute ML, Weaver AL, et al. Matched comparison of radical nephrectomy vs nephron-sparing surgery in patients with unilateral renal cell carcinoma and a normal contralateral kidney. Mayo Clin Proc 2000;75:1236-42. http://dx.doi.org/10.4065/75.12.1236

11. Miller DC, Hollingsworth JM, Hafez KS, et al. Partial nephrectomy for small renal masses: An emerging qual ity of care concern? J Urol 2006;175:853-7. http://dx.doi.org/10.1016/S0022-5347 (05)00422-2

12. Levey AS, Coresh J, Greene T, et al. Chronic Kidney Disease Epidemiology Collaboration. Using standardized serum creatinine values in the modification of diet in renal disease study equation for estimating glomerular filtration rate. Ann Intern Med 2006; 145:247-54.

13. Dindo D, Demartines N, Clavien PA. Classification of Surgical Complications. Ann Surg 2004;240:205-13. hittp://dx.doi.org/10.1097/01.sla.0000133083.54934.ae

14. National Kidney Foundation (NKF) Kidney Disease Outcome Quality Initiative (K/DOOI) advisory board. Clinical practice guidelines for chronic kidney disease: evaluation, classification, and stratification. Am J Kidney Dis 2002;39:S65.

15. Sarnak MJ, Levey AS, Hamm LL, et al. Kidney disease as a risk factor for development of cardiovascular disease. Circulation 2003;108:2154-69. http://dx.doi.org/10.1161/01.CIR.0000095676.90936.80

16. Go AS, Chertow GM, Fan G, et al. Chronic kidney disease and the risks of death, cardiovascular events, and hospitalization. New Engl J Med 2004;351:1296-305. http://dx.doi.org/10.1056/NEJMoo041031

17. Huang WC, Elkin EB, Levey AS, et al. Partial nephrectomy versus radical nephrectomy in patients with small renal tumours-ss there a difference in mortality and cardiovascular outcomes? I Urol 2009;181:55-61. hittp://dx.doi.org/10.1016/i.juro.2008.09.017

18. Campbell SC, Novick AC, Belldegrun A, et al. Guideline for Management of the clinical TI Renal Mass. J Urol 2009;182:1271-9. httrp://dx.doi.org/10.1016/i.juro.2009.07.004

19. Gill IS, Kavoussi LR, Lane BR, et al. Comparison of 1,800 Laparoscopic and Open Partial Nephrectomies for Single Renal Tumors. J Urol 2007;178:41-6. http://dx.doi.org/10.1016/i.juro.2007.03.038

20. Patard JJ, Pantuck AJ, Crepel $M$, et al. Morbidity and clinical outcome of nephron-sparing surgery in relation to tumour size and indication. Eur Urol 2007;52:148-54. http://dx.doi.org/10.1016/i. eururo.2007.01.039

21. Porpiglia $\mathrm{F}$, Volpe $\mathrm{A}$, Billia $\mathrm{M}$, et al. Laparoscopic versus open partial nephrectomy: analysis of the current literature. Eur Urol 2008;53:732-42. hitp://dx.doi.org/10.1016/i.eururo.2008.01.025

22. Lane BR, Gill IS. 5-Year outcomes of laparoscopic partial nephrectomy. J Urol 2007; 177:70-4. hitp:// dx.doi.org/10.1016/i.juro.2006.08.093

Correspondence: Dr. Naii J. Touma, Kingston General Hospital, 76 Stuart St., Kingston, ON K7L 2V1; nitouma@gmail.com 